\title{
USING AugMeNTEd REALITY AND HolOgRAPHIC TECHNOLOGY IN AN INTRODUCTORY COURSE ON DATA STRUCTURES AND Algorithms
}

\author{
Igor Ivkovic ${ }^{1}$ and Sage Franch ${ }^{2}$ \\ ${ }^{1}$ Systems Design Engineering, University of Waterloo; ${ }^{2}$ Microsoft Canada \\ igor.ivkovic@uwaterloo.ca, sage.franch@microsoft.com
}

\begin{abstract}
Augmented reality (AR) technology facilitates augmentation of current views with digital artifacts, such as information, three-dimensional objects, audio, and video. Mixed reality (MR) represents an enhanced version of $A R$, where advanced spatial mapping is used to anchor digital artifacts in physical space. Using MR technology, digital artifacts can be more closely integrated into the natural environment, thereby transcending physical limitations and creating enhanced blended learning environments. In this paper, we propose an approach for integration of MR technology into engineering education. Specifically, we propose to integrate Microsoft HoloLens into a first-year course on data structures and algorithms to improve student engagement and learning outcomes. In the pilot study, students were assigned to implement $A^{*}$ algorithm and then given a chance to visualize their implementation using Microsoft HoloLens. The feedback provided by students indicated increased engagement and interest in graph-based path-finding algorithms as well as MR technology.
\end{abstract}

Keywords: Engineering Education, Enhancing Learning Experience, Incorporating New Technology into Teaching, Using Mixed Reality in Engineering Education, Expanding Teaching Capabilities

\section{INTRODUCTION}

Augmented reality (AR) represents computer-based technology and devices that facilitate augmentation of current views with digital artifacts, such as information, three-dimensional objects, audio, and video [1]. Students have recently been exposed to the Pokémon Go video game [2], which is an example of AR use in practice. Mixed Reality (MR) represents an enhanced version of AR, where advanced spatial mapping is used to anchor digital artifacts in physical space; this enables digital elements to not only overlay the physical world, but interact with physical objects and respond to changes in physical space [3].

One of the strengths of Mixed Reality is the hybridization of two physical realities by bringing them together with virtual renderings. This has been investigated by Microsoft Research and Holoportation project that uses HoloLens to virtually bring users in separate places together in one hybrid space [4].

Using MR technology, digital artifacts can be more closely integrated into the natural environment, transcending physical limitations and creating enhanced blended learning environments. One example of MR technology is the Microsoft HoloLens platform, which is an untethered headset that provides spatial, visual, and auditory rendering of programmable, dynamic holographic objects [5].

In this paper, we propose an approach for integration of MR technology into undergraduate engineering curriculum. Specifically, we propose to integrate the Microsoft HoloLens platform into a first-year course on data structures and algorithms (DSA) to improve student engagement and learning outcomes.

In the pilot study, administered in Winter 2017, students were assigned to implement $\mathrm{A}^{*}$ algorithm and then given a chance to visualize their implementation using Microsoft HoloLens. The feedback provided by students indicated increased engagement and interest in graph-based path-finding algorithms as well as MR technology.

The rest of this paper is organized as follows: in Section 2, we describe problem context and previous state of the course in which the technology was applied, explain the reasoning for curriculum update, go through the objectives of the pilot study, and explain the results of the experiment. Finally, in Section 3, we provide summary and conclusions, and explain what changes are planned for the next iteration

\section{INTRODUCING MIXED REALITY INTO ENGINEERING EDUCATION}

Traditionally, data structures and algorithms represent a conceptual field - students are dealing with algorithmic code that produces optimized output according to given specifications. The use of an MR platform as a testbed for students' code is a promising enhancement to the course learning objectives - it is about making the concepts come alive for the students. 


\subsection{Problem Context: A First-Year Course on Data Structures and Algorithms}

The context of this study is an introductory, first-year course on data structures and algorithms for engineering students. In this course, among other topics, students are taught the basics of graphs as data structures composed of nodes and edges. Students learn how to use graphs as underlying data structures to solve practical problem scenarios, represent graphs using matrices or linked lists, traverse graphs using depth-first and breadth-first approaches, and apply shortest-path algorithms to find shortest paths between graph nodes.

In the previous versions of the course, based on current curriculum objectives, students were taught Dijkstra's algorithm as a single-source, shortest-path algorithm that finds shortest paths from one starting node to all other nodes in a weighted, directed graph where all the weights are non-negative values.

One method that was used to assess student understanding of Dijkstra's algorithm and its steps included assigning students to apply the algorithm to a given graph model and trace its steps. For example, Dijkstra's algorithm can be applied to the graph model given in Figure 1 to find all the shortest paths starting from node 2; the illustration of this particular algorithm application is provided below.

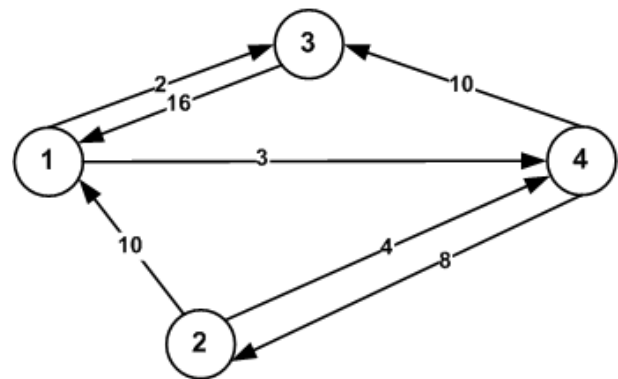

Figure 1. Sample Directed Weighted Graph

At each iteration, Dijkstra's algorithm computes the cost of the paths that go through the current node to all accessible nodes. If a shorter path to a node is found, then the distance to that node is updated and the found path stored.

- $\quad$ [Iteration: 1] Visited Set $=\{2\}$, Unvisited Set $=\{1,3,4\}$, Current Node $(C)=2$, Distance to $C\left(d_{C}\right)=0$.

o Distance to Node $1\left(\mathrm{~d}_{1}\right)=10$, Path to (1): (2)-(1), Distance to Node $2\left(\mathrm{~d}_{2}\right)=0$, Path to (2): (2)-(2), Distance to Node $3\left(\mathrm{~d}_{3}\right)=\infty$, Path to (3): Unknown, Distance to Node $4\left(\mathrm{~d}_{4}\right)=4$, Path to (4): (2)-(4).

- $\quad$ [Iteration: 2] Visited Set $=\{2,4\}$, Unvisited Set $=\{1,3\}$, Current Node $(C)=4$, Distance to $C\left(d_{C}\right)=4$. o Distance to Node $1\left(\mathrm{~d}_{1}\right)=10$, Path to (1): (2)-(1), Distance to Node $2\left(\mathrm{~d}_{2}\right)=0$, Path to (2): (2)-(2), Distance to Node $3\left(d_{3}\right)=14$, Path to (3): (2)-(4)-(3), Distance to Node $4\left(\mathrm{~d}_{4}\right)=4$, Path to (4): (2)-(4).

- $\quad$ IIteration: 3] Visited Set $=\{1,2,4\}$, Unvisited Set $=\{3\}$, Current Node $(C)=1$, Distance to $C\left(\mathrm{~d}_{\mathrm{C}}\right)=10$.

o Distance to Node $1\left(\mathrm{~d}_{1}\right)=10$, Path to (1): (2)-(1), Distance to Node $2\left(\mathrm{~d}_{2}\right)=0$, Path to (2): (2)-(2), Distance to Node $3\left(d_{3}\right)=12$, Path to (3): (2)-(1)-(3), Distance to Node $4\left(\mathrm{~d}_{4}\right)=4$, Path to (4): (2)-(4).

- $\quad$ IIteration: 4] Visited Set $=\{1,2,3,4\}$, Unvisited Set $=\{\}$, Current Node $(C)=3$, Distance to $C\left(d_{C}\right)=12$.

o Distance to Node $1\left(\mathrm{~d}_{1}\right)=10$, Path to (1): (2)-(1), Distance to Node $2\left(\mathrm{~d}_{2}\right)=0$, Path to (2): (2)-(2), Distance to Node $3\left(d_{3}\right)=12$, Path to (3): (2)-(1)-(3), Distance to Node $4\left(\mathrm{~d}_{4}\right)=4$, Path to (4): (2)-(4).

- [Iteration: 5] No more unvisited nodes, so output the results and terminate.

\subsection{Continuous Course Improvement: Introducing A* Algorithm}

As part of continuous curriculum improvement and based on student interest, other graph-based algorithms were considered to be included in the course. For navigation and path finding in physical space where coordinates are available for individual nodes, there may be more efficient versions of the Dijkstra's algorithm available that allow for the use of navigational heuristics to improve performance.

One such algorithm that was considered is $\mathrm{A}^{*}$ algorithm [6], which is a heuristics-based path-finding algorithm, and can be viewed as refinement of Dijkstra's algorithm. With $\mathrm{A}^{*}$, starting and ending points are provided, and then the algorithm makes use of heuristic costs to find the shortest path between the given points.

Possible heuristics include, but are not limited to, Euclidian distance, where heuristic cost

$$
\text { hCost }=\sqrt{(\text { node. } x-\operatorname{target} . x)^{2}+(\text { node. } y-\operatorname{target} y)^{2}} ;
$$

and Manhattan distance, where heuristic cost

$$
\text { hCost }=\mid \text { node. } x-\operatorname{target} . x|+| \text { node. } y-\operatorname{target} . y \mid \text {. }
$$

In the above expressions, node. $x$ and node. $y$ are the coordinates of the considered graph nodes, and target. $x$ and target. $y$ are the coordinates of the target/ending node.

One method that may be used to assess student understanding of $\mathrm{A}^{*}$ algorithm includes assigning students to apply the algorithm to a given world state, and trace its steps. For example, A* algorithm can be applied 
to the graph model given in Figure 2 to find the shortest path from $(0,0)$ to $(1,3) ;(x, y)$ coordinates are given in each cell; $\mathrm{T}$ indicates that a cell is walkable and $\mathrm{F}$ indicates that a cell is not walkable.

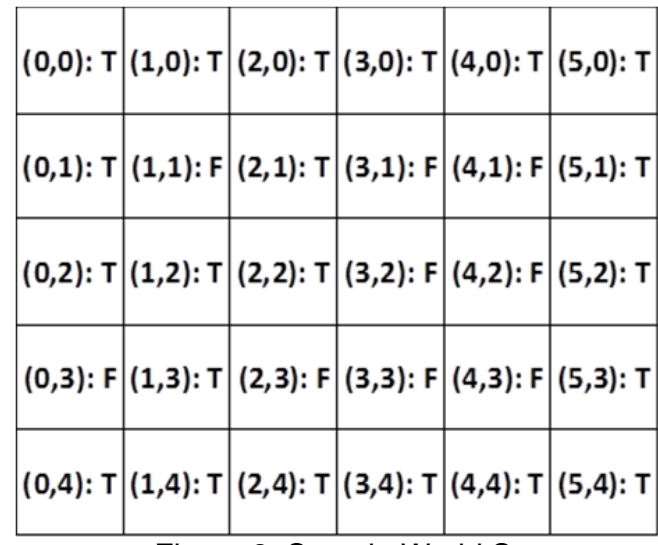

Figure 2. Sample World State

Illustration of $\mathrm{A}^{*}$ application using Manhattan distance, where only straight paths are allowed, is provided below. At each step, a node with the lowest fCost is selected from the Open Set and moved to the Closed Set; all of the selected node's neighbours that are walkable are then included or updated in the Open Set.

Some details, such as step-by-step computation of gCost, hCost, and fCost, and keeping track of parent nodes for each node, were omitted for brevity.

- $\quad$ [Initialization] Closed Set $=\{\}$, Open Set $=$

$\{[(\mathrm{x}, \mathrm{y})=(0,0), \mathrm{gCost}=0, \mathrm{hCost}=4, \mathrm{fCost}=4]\}$

- $\quad$ [Iteration: 1$]$ Closed Set $=$

$\{[(\mathrm{x}, \mathrm{y})=(0,0), \mathrm{gCost}=0, \mathrm{hCost}=4$, fCost $=4]\}$, Open Set $=$

$\{[(\mathrm{x}, \mathrm{y})=(0,1), \mathrm{gCost}=1, \mathrm{hCost}=3$, fCost $=4]$,

$[(\mathrm{x}, \mathrm{y})=(1,0), \mathrm{gCost}=1, \mathrm{hCost}=3$, fCost $=4]\}$

- $\quad$ [Iteration: 2] Closed Set $=$

$\{[(\mathrm{x}, \mathrm{y})=(0,0), \mathrm{gCost}=0, \mathrm{hCost}=4, \mathrm{fCost}=4]$, $[(\mathrm{x}, \mathrm{y})=(0,1), \mathrm{gCost}=1, \mathrm{hCost}=3$, fCost $=4]\}$, Open Set $=$

$\{[(\mathrm{x}, \mathrm{y})=(0,2), \mathrm{gCost}=2$, hCost $=2, \mathrm{fCost}=4]$,

$[(\mathrm{x}, \mathrm{y})=(1,0), \mathrm{gCost}=1, \mathrm{hCost}=3$, fCost $=4]\}$

- $\quad$ [Iteration: 3$]$ Closed Set =

$\{[(\mathrm{x}, \mathrm{y})=(0,0), \mathrm{gCost}=0, \mathrm{hCost}=4$, fCost $=4]$,

$[(\mathrm{x}, \mathrm{y})=(0,1), \mathrm{gCost}=1, \mathrm{hCost}=3, \mathrm{fCost}=4]$,

$[(\mathrm{x}, \mathrm{y})=(0,2), \mathrm{gCost}=2, \mathrm{hCost}=2, \mathrm{fCost}=4]\}$,

Open Set $=$

$\{[(\mathrm{x}, \mathrm{y})=(1,2), \mathrm{gCost}=3, \mathrm{hCost}=1, \mathrm{fCost}=4]$,

$[(\mathrm{x}, \mathrm{y})=(1,0), \mathrm{gCost}=1, \mathrm{hCost}=3$, fCost $=4]\}$
- $\quad$ [Iteration: 4] Closed Set =

$\{[(\mathrm{x}, \mathrm{y})=(0,0), \mathrm{gCost}=0, \mathrm{hCost}=4, \mathrm{fCost}=4]$,

$[(\mathrm{x}, \mathrm{y})=(0,1), \mathrm{gCost}=1, \mathrm{hCost}=3, \mathrm{fCost}=4]$,

$[(\mathrm{x}, \mathrm{y})=(0,2), \mathrm{gCost}=2, \mathrm{hCost}=2, \mathrm{fCost}=4]$,

$[(\mathrm{x}, \mathrm{y})=(1,2), \mathrm{gCost}=3, \mathrm{hCost}=1, \mathrm{fCost}=4]\}$,

Open Set $=$

$\{[(\mathrm{x}, \mathrm{y})=(1,3), \mathrm{gCost}=4, \mathrm{hCost}=0, \mathrm{fCost}=4]$,

$[(\mathrm{x}, \mathrm{y})=(2,2), \mathrm{gCost}=4, \mathrm{hCost}=2$, fCost $=6]$,

$[(\mathrm{x}, \mathrm{y})=(1,0), \mathrm{gCost}=1, \mathrm{hCost}=3$, fCost $=4]\}$

- $\quad$ [Iteration: 5] Closed Set =

$\{[(\mathrm{x}, \mathrm{y})=(0,0), \mathrm{gCost}=0, \mathrm{hCost}=4, \mathrm{fCost}=4]$,

$[(\mathrm{x}, \mathrm{y})=(0,1), \mathrm{gCost}=1, \mathrm{hCost}=3$, fCost $=4]$,

$[(\mathrm{x}, \mathrm{y})=(0,2), \mathrm{gCost}=2, \mathrm{hCost}=2, \mathrm{fCost}=4]$,

$[(x, y)=(1,2)$, gCost $=3$, hCost $=1$, fCost $=4]$,

$[(\mathrm{x}, \mathrm{y})=(1,3), \mathrm{gCost}=4, \mathrm{hCost}=0, \mathrm{fCost}=4]\}$,

Target node $(1,3)$ found, so output the shortest path and terminate.

As shown above, the application of $A^{*}$ algorithm requires students to follow complex algorithmic steps that include the use of heuristics and consistent updating of multiple data items and data sets.

Before a decision was made to make $A^{*}$ algorithm a part of the course curriculum in a first-year course, approaches to improving student engagement, simplifying algorithm application, and improving learning outcomes, were considered.

One such venue that was considered was the use of technology with which students may be already familiar and which in turn may demonstrate the application of $\mathrm{A}^{*}$ algorithm in practice. Based on a successful application of AR technology to improve learning outcomes in a course on graphical communication, AR technology was considered as a suitable technology for this course.

\subsection{Pilot Study: Using MR to Improve Student Engagement and Learning Outcomes}

Both AR and MR technologies may offer opportunities for enhancement of engineering education, such as allowing students to experiment with concepts before idea maturization. These technologies may also allow students to visualize and interact with environments that previously would have been difficult, expensive, or even impossible to access; for example, interaction with complex machinery, problem-solving scenarios for mission-critical systems, or simulation of environments that are not physically possible on Earth.

MR technology was considered as an enhancement of AR solutions, where augmented reality is enhanced through the use of holographic technology and advanced spatial mapping. 
The advantage of MR over AR is in the anchoring and interaction between physical and virtual objects. Given that beacons can be anchored in physical space, students can move freely about the space and observe and interact with the virtual artifacts as though they were physical. Virtual Reality (VR) has a similar effect, but places the observer in an entirely digital environment rather than placing digital artifacts in the observer's natural environment. We propose that this MR visualization of traditionally abstract concepts in environments comfortable to the user may help student learning.

As the target platform for the study, we selected Microsoft HoloLens as a state-of-the-art MR platform [5]. VR platforms were also considered, such as Oculus Rift and Google Cardboard, and may be included in future iterations of this study.

With respect to the context of the study, we focused on the following steps:

1. Have students implement $A^{*}$ algorithm as a course assignment;

2. Develop a holographic application that demonstrates the use of $A^{*}$ path-finding in a virtual environment; and

3. Have students visualize their implementation through the use of the HoloLens platform.

For Step 1, students implemented an assignment as part of the course curriculum, but expressed difficulty visualizing specific steps.

For Step 2, a small team of research assistants helped us implement a holographic application that demonstrated the use of $\mathrm{A}^{*}$ algorithm. The implementation was performed using HoloLens SDK and related tools.

For Step 3, students were provided an opportunity to use the app to visualize steps of algorithm application through an in-class demo. The demo allowed them to:

- Physically enter the target world, and interact with holographic objects;

- Move holographic obstacles and create challenging paths for the algorithm to solve;

- Issue commands using voice or hand gestures;

- Observe a holographic object automatically move from the start point to the end point based on computed shortest path; and

- Inspect distance costs of points on the movement path to better understand algorithm application.

\subsection{Preliminary Experiment Results}

The pilot study was applied in Winter 2017. Approximately 108 students were invited to participate in the demonstration. Some preliminary data was collected but has not been conclusively analyzed as of yet.
General feedback provided by students indicated increased engagement and interest in graph-based pathfinding algorithms as well as MR technology.

\section{SUMMARY AND CONCLUSIONS}

MR technology offers enhancements over AR solutions where augmented reality is enhanced through the use of holographic technology and advanced spatial mapping. In this paper, we presented the steps for integration of MR technology into a first-year course on data structures and algorithms. The approach will be applied again in Spring 2017.

In future iterations of the approach, we will include further data collection and validation, alignment of the approach with the intended learning outcomes of the course, and enhancement of the HoloLens visualization based on received feedback.

\section{Acknowledgements}

We thank Engineering Ideas Clinic, Microsoft University Relations, and Microsoft Corporation, for their kind contribution of HoloLens kits that made the pilot implementation possible.

We thank Dr. Carolyn MacGregor, and other colleagues from Systems Design Engineering, Mechatronics Engineering, and Faculty of Engineering, for their contribution to this study. We also thank Clement Yuen, and other undergraduate and graduate students for their time and effort.

\section{References}

[1] Borko Furht (Ed), Handbook of Augmented Reality, Springer 2011.

[2] Nick Wingfield and Mike Isaac, Pokémon Go Brings Augmented Reality to a Mass Audience, The New York Times, July 11, 2016.

[3] Enrico Costanza, Andreas Kunz, and Morten Fjeld, "Mixed Reality: A Survey”, Human Machine Interaction, LNCS 5440, pp. 47-68, 2009.

[4] Henry Chen, Austin S. Lee, Mark Swift, and John C. Tang, "3D Collaboration Method over HoloLens ${ }^{\mathrm{TM}}$ and Skype ${ }^{\mathrm{TM}}$ End Points", $3^{\text {rd }}$ Intl Workshop on Immersive Media Experiences (ImmersiveME '15), ACM, New York, USA, 27-30, 2015.

[5] Microsoft Corporation, HoloLens Overview, 2017. [Online]. http://www.microsoft.com/en-us/hololens

[6] Amit Patel, Introduction to A*, From Amit's Thoughts on Pathfinding, 2017. [Online].

http://theory.stanford.edu/ amitp/GameProgramming/AStarCom parison.html 